\title{
Modernização de Arquiteturas de Sistemas para suporte à Transformação Digital
}

\author{
Pablo Luiz Leon ${ }^{1}$, Flávio Horita ${ }^{1}$ \\ ${ }^{1}$ Centro de Matemática, Computação e Cognição (CMCC) \\ Universidade Federal do ABC (UFABC) \\ Av. dos Estados, 5001 - Bangú 09210-580 Santo André - SP - Brazil \\ \{pablo.leon, flavio.horita\}@ufabc.edu.br
}

\begin{abstract}
With the widespread use of technology, companies and the government have used technologies to enable the digital transformation of products and services demanded by society. However, the decentralization of systems becomes important to create new solutions and / or services for users with a more connected and real-time experience. There are two challenges: 1) how to decentralize existing legacy systems to provide a technology solution that meets the new needs of users in this more digital society; and 2) how to create a systems architecture that addresses the characteristics inherent in digital transformation. In this sense, this project proposal aims to present an approach to modernize legacy systems in order to converge business in a digital transformation scenario.
\end{abstract}

Resumo. Com a massificação no uso da tecnologia, empresas e o governo têm empregado tecnologias para viabilizar a transformação digital dos produtos $e$ serviços demandados pela sociedade. Contudo, a descentralização dos sistemas torna-se importante para criar novas soluções elou serviços aos usuários com uma experiência mais conectada e em tempo real. Há dois desafios: 1) como descentralizar os sistemas legados existentes para fornecer uma solução de tecnologia que atenda as novas necessidades dos usuários desta sociedade mais digital; e 2) como criar uma arquitetura de sistemas que contemple as características inerentes na transformação digital. Neste sentido, esta proposta de projeto busca apresentar uma abordagem de modernizaçãoo de sistemas legados de maneira à convergir os negócios um cenário de transformação digital.

\section{Introdução}

A necessidade das empresas e/ou governo de possuir soluções de tecnologias da informação que sejam escaláveis e de fácil manutenção nos dias atuais é fundamental para o sucesso dos modelos de negócios.

A transformação digital tem papel fundamental neste cenário. Isso porque ela pavimenta o caminho das empresas, ajudando vários setores, aprimorando, estendendo e redefinindo seus produtos e serviços físicos ou tradicionais por meio de conteúdo digital, remodelando as proposições de valor entregues a seus clientes e criando novos fluxos de receita para garantir sua sobrevivência nos mercados [Karimi and Walter 2015] e [Berman et al. 2011]. 
Assim, conforme é citado por [Maier 1996, Inocêncio et al. 2019] sobre os Sistemas-de-Sistemas (SoS), onde eles possuem caraterística de integrar sistemas e são independentes, cada um com seus próprios propósitos e funções. Quando interagem com os demais sistemas, visam cumprir uma missão global. Isso torna o mundo mais conectado digitalmente, gerando uma demanda grande de integrar soluções de TI para o negócio.

Diante disto, torna-se importante ter uma alternativa de criar modelos arquiteturais de fácil manutenção, baixa acoplamento e com escalabilidade. Em outras palavras, estas empresas precisam "modernizar" suas aplicações. A literatura e prática evolui consideravelmente nesta temática [Gouigoux and Tamzalit 2017]. Por exemplo, [Furda et al. 2017] propõe uma abordagem onde o sistema legado (monólito) é quebrado em partes para a construção de micro serviços que juntos proveem a mesma solução, porém são escaláveis, de fácil manutenção e permitem as empresas entregar valor. Outra linha de trabalhos emprega o uso de técnicas de modelagem de contexto de negócio para separação de grandes sistemas em micro serviços ou técnicas de análises dos dados para a separação de funcionalidades de um sistema maior em micro serviços, onde ambos podem conviver na mesma arquitetura [Levcovitz et al. 2016]. A abordagem de recorte dos contextos de negócio no sistema legado é importante. Isso porque ela permite definir onde poderiam acontecer a criação de micro serviços. A execução dessa estratégia pode melhorar o tempo de resposta das áreas de tecnologia e fornecer serviços robustos, escaláveis e de fácil manutenção para o negócio convergindo para esse contexto de transformação digital [Rademacher et al. 2018, Jamshidi et al. 2018].

Neste cenário, este trabalho busca apresentar uma abordagem de modernização de sistemas baseado em um modelo de arquitetura que permita a convivência entre sistema legado e sistema moderno. Para isto, é apresentando o uso DDD (Domain Drive Design) como alternativa para permitir o reconhecimento de contextos das funcionalidades e efetuar o recorte de código no ambiente legado, modernizando o mesmo para microsserviços. Essa ação irá gerar aderência a necessidade de baixo acoplamento, fácil manutenção e escalabilidade dos sistemas, alinhada a transformação digital e as necessidades e prioridades do negócio.

\section{Apresentação do Problema}

O surgimento de novas tecnologias como Big Data, Inteligência Artificial, processamento distribuídos e orientação a eventos, junto com o advento e viabilidade de produtos e serviços digitais, gera uma força de transformação dos negócios [Vermesan and Bacquet 2017]. Ao caminhar nessa direção as empresas podem entrar em uma nova era, a chamada Era Digital, ou passar a sofrer alterações em suas estruturas dada a transformação digital [Singh and Hess 2017]. Isso porque todas as informações passam a oferecer produtos e/ou serviços aos clientes estão contidas nesse ambiente (Internet). De um lado, há empresas que estão nascendo para fornecer esses produtos e serviços e competir no mercado com sistemas modernos que já nascem com essas características para sobreviverem nesse meio. Por outro lado, existem empresas com anos de existência, mas precisam continuar competitivas no mercado.

Surge então a necessidade de modernizar seus sistemas, oferecendo integrações, compartilhamento de informações, e uma velocidade maior na atualizações dos seus pro- 
dutos e serviços [Desmet et al. 2016].

\section{Proposta de Solução}

A literatura existente na área tem empregado a arquitetura de microsserviços para realizar a modernização dos sistemas. Os microsserviços são uma peça fundamental de um modelo arquitetural que visa gerar flexibilização de ambientes. Eles possibilitem a convivência com sistemas monolíticos (antigos) [Fritzsch et al. 2019, Computing 2017]. Essa arquitetura de convivência pode habilitar negócios a estarem aderentes aos anseios da transformação digital [Choudhary et al. 2019].

Nesse contexto, faz-se também necessário efetuar a distribuição desses componentes de forma rápida, simples e automática. Para tal, temos o modelo de cloud computing que permite a criação de aplicativos modernos por meio do provisionamento de hardware sob demanda de forma rápida [Viggiato et al. 2018] [Gkikopoulos 2019, Dragičević and Bošnjak 2019]. Essa necessidade que a transformação digital traz para as empresas, se transformam em novos desafios para a engenharia de software [Fritzsch et al. 2019].

Pesquisas recentes mostram a necessidade da adoção ou a convergência das empresas em adotar esse tipo de estrutura de aplicação na web modernas para conseguir estarem prontas na era digital [Bogner et al. 2019].

Com o uso da Internet e da mobilidade digital, existe uma demanda em se criar sistemas que são capazes de ser resilientes e escaláveis. A arquitetura de microsserviços é feita com esse propósito [Chawla and Kathuria 2019]. Desta forma, a engenharia de software precisa adotar modelos de arquitetura ágeis capazes de entregar software de forma ágil para o negócio.

Modernizar sistemas e deixa-los em modelos de infraestrutura antigos não irá torna-los fáceis de mantê-los [Linthicum 2016]. A cloud computing é a estratégia mais rápida e eficiente de provisionar computação para processamento dos negócios digitais. A ligação entre o software construído e a sua utilização de forma rápida agrega o conceito de DevOps que é um mecanismo catalizador de criação de software com provisionamento de capacidade computacional [Wiedemann et al. 2019].

Diante deste cenário, este trabalho apresenta uma abordagem de modernização de sistemas baseado em um modelo de arquitetura para a convivência entre sistemas monolíticos e modernos, i.e., com componentes de software de fácil escalabilidade, manutenção e baixa complexidade (microsserviços). Utilizado DDD como ferramenta no recorte das funções no ambiente monolítico e efetuando a criação de serviços modernos. Assim, busca-se gerar uma aderência a transformação digital e as necessidades do negócio em priorizar o que é de maior valor para a empresa no momento.

\section{Projeto de Avaliação da Solução}

Serão empregadas técnicas e instrumentos para a avaliação das abordagens desenvolvidas neste projeto em um estudo de caso centrado no setor financeiro. Entrevistas semi-estruturadas e grupos focais serão conduzidos com membros da empresa conforme [Seaman 1999] e [Nili et al. 2014]. Isto tem como objetivo levantar os dados para estruturar o estudo de caso, bem como identificar as abordagens existentes na empresa para a 
modernização de sistemas. Alguns exemplos de dados: (Tipos de arquiteturas de software existentes, quantas funcionalidades em média por unidade de software, qual o tempo indisponível médio de sistemas monolíticos e sistemas baseados em microsserviços?, qual é o tempo médio de manutenção de sistemas monolíticos e sistemas baseados em micro serviços?). Além disso, será realizada uma análise qualitativa de documentos associados ao caso de estudo. Por fim, também poderá ser realizada a implementação de um sistema e/ou uma simulação de sistemas que reflita a abordagem de modernização do sistema; por exemplo, empregando a abordagem apresentada em [Neto et al. 2017].

\section{Atividades já realizadas}

Abaixo a lista de atividades já feitas para essa pesquisa:

1 Leitura de artigos com proximidade ao tema;

2 Definição da pesquisa, hipótese levantada, escopo preliminar da dissertação;

3 Pesquisa em base de conhecimento usando palavras chave como: (modernization, digital transformation, monolitih, system modernization);

4 Pesquisa de artigos sobre revisão sistemática em sistemas de informação;

5 Definição de palavras chave para efetuar revisão sistemática;

6 Definição de Critérios de inclusão e exclusão de artigos;

7 Ajuste fino de String para pesquisar em bases de conhecimento;

8 Execução de String em 4 bases de conhecimento;

9 Leitura de aproximadamente 347 artigos para aplicar critérios de inclusão e exclusão;

10 Inicio da escrita da Revisão Sistemática.

\section{Considerações Finais}

Este trabalho apresentou a proposta para a criação de um modelo de modernização de sistemas através do uso de arquiteturas de SoS bem como características que permeiam a transformação digital para criação de artefatos de engenharia de software que desenhe a arquitetura dos sistemas e evidencie na arquitetura dos sistemas os requisitos funcionais e não funcionais de um contexto do problema. Tal estudo, envolve o conhecimento de diferentes abordagens de analise de Requisitos para desenho de arquiteturas sistêmicas e conhecimentos de pilares de transformação digital. Além das análises, o projeto prevê a disponibilização de um conjunto de artefatos ou roteiro que auxilie o arquiteto na concepção de soluções aderentes a transformação digital. O objetivo geral é contribuir para a academia e indústria ligada ao desenvolvimento de sistemas, fornecendo métodos e guias com informações relevantes que norteiam a criação desse eco sistema de integração entre sistemas.

Como limitações e riscos, é possível citar: (1) o tempo de estudo, pois há uma infinidade de artefatos e arquiteturas existentes, dificultando a definição do método; (2) a validação do método em mais de uma empresa; e (3) a política da empresa a qual é alvo de pesquisa na aceitação da divulgação dos resultados da pesquisa.

\section{Agradecimentos}

Os autores agradecem ao apoio financeiro do Conselho Nacional de Desenvolvimento Científico e Tecnológico (CNPq), Processo Nro 437937/2018-6. 


\section{Referências}

Berman, S. J., Bell, R., et al. (2011). Digital transformation: Creating new business models where digital meets physical. IBM Institute for Business Value, pages 1-17.

Bogner, J., Fritzsch, J., Wagner, S., and Zimmermann, A. (2019). Microservices in Industry: Insights into Technologies, Characteristics, and Software Quality. Proceedings 2019 IEEE International Conference on Software Architecture - Companion, ICSA-C 2019, (March):187-195.

Chawla, H. and Kathuria, H. (2019). Building Microservices Applications on Microsoft Azure. Building Microservices Applications on Microsoft Azure, pages 1-20.

Choudhary, S., Thomas, I., Bahrami, M., and Sumioka, M. (2019). Accelerating the digital transformation of business and society through composite business ecosystems. In International Conference on Advanced Information Networking and Applications, pages 419-430. Springer.

Computing, S. J. (2017). AN OVERVIEW OF SELF-ADAPTIVE TECHNIQUES. 11(2):115-136.

Desmet, D., Loffler, M., and Weinberg, A. (2016). Modernizing it for a digital era. McKinsey, September.

Dragičević, Z. and Bošnjak, S. (2019). Agile architecture in the digital era: Trends and practices. Strategic Management, 24(2):12-33.

Fritzsch, J., Bogner, J., Wagner, S., and Zimmermann, A. (2019). Microservices Migration in Industry: Intentions, Strategies, and Challenges. 2019 IEEE International Conference on Software Maintenance and Evolution (ICSME), pages 481-490.

Furda, A., Fidge, C., Zimmermann, O., Kelly, W., and Barros, A. (2017). Migrating enterprise legacy source code to microservices: on multitenancy, statefulness, and data consistency. IEEE Software, 35(3):63-72.

Gkikopoulos, P. (2019). Data distribution and exploitation in a global microservice artefact observatory. In 2019 IEEE World Congress on Services (SERVICES), volume 2642, pages 319-322. IEEE.

Gouigoux, J. and Tamzalit, D. (2017). From monolith to microservices: Lessons learned on an industrial migration to a web oriented architecture. In 2017 IEEE International Conference on Software Architecture Workshops (ICSAW), pages 62-65.

Inocêncio, T. J., Gonzales, G. R., Cavalcante, E., and Horita, F. E. (2019). Emergent behavior in system-of-systems: A systematic mapping study. In Proceedings of the XXXIII Brazilian Symposium on Software Engineering, pages 140-149.

Jamshidi, P., Pahl, C., Mendonça, N. C., Lewis, J., and Tilkov, S. (2018). Microservices: The journey so far and challenges ahead. IEEE Software, 35(3):24-35.

Karimi, J. and Walter, Z. (2015). The role of dynamic capabilities in responding to digital disruption: A factor-based study of the newspaper industry. Journal of Management Information Systems, 32(1):39-81.

Levcovitz, A., Terra, R., and Valente, M. T. (2016). Towards a technique for extracting microservices from monolithic enterprise systems. arXiv preprint arXiv:1605.03175. 
Linthicum, D. S. (2016). Practical use of microservices in moving workloads to the cloud. IEEE Cloud Computing, 3(5):6-9.

Maier, M. W. (1996). Architecting principles for systems-of-systems. INCOSE International Symposium, 6(1):565-573.

Neto, V. V. G., Paes, C. E. B., Garcés, L., Guessi, M., Manzano, W., Oquendo, F., and Nakagawa, E. Y. (2017). Stimuli-sos: a model-based approach to derive stimuli generators for simulations of systems-of-systems software architectures. Journal of the Brazilian Computer Society, 23(1):13.

Nili, A., Tate, M., Johnstone, D., and Gable, G. G. (2014). A framework for qualitative analysis of focus group data in information systems. In Proceedings of the $25^{\text {th }}$ Australasian Conference on Information Systems, Auckland, New Zealand.

Rademacher, F., Sorgalla, J., and Sachweh, S. (2018). Challenges of domain-driven microservice design: A model-driven perspective. IEEE Software, 35(03):36-43.

Seaman, C. B. (1999). Qualitative methods in empirical studies of software engineering. IEEE Transactions on Software Engeneering, 25(3):1-16.

Singh, A. and Hess, T. (2017). How chief digital officers promote the digital transformation of their companies. MIS Quarterly Executive, 16(1).

Vermesan, O. and Bacquet, J. (2017). Cognitive Hyperconnected Digital Transformation: Internet of Things Intelligence Evolution. River Publishers.

Viggiato, M., Terra, R., Rocha, H., Valente, M. T., and Figueiredo, E. (2018). Microservices in practice: A survey study. arXiv preprint arXiv:1808.04836.

Wiedemann, A., Forsgren, N., Wiesche, M., Gewald, H., and Krcmar, H. (2019). The DevOps phenomenon. Quеие, 17(2):1-20. 